\title{
A Provisional Model for the Optimal Management of a Charging Station Assisted by Photovoltaic Panels for Plug-In Electric Vehicles
}

\author{
Rizzo Gianfranco ${ }^{1, *}$, Tiano Francesco Antonio ${ }^{2}$ and Marino Matteo ${ }^{3}$ \\ ${ }^{1}$ Full Professor at Dpt. of Industrial Engineering, University of Salerno, Fisciano, Salerno, Italy \\ ${ }^{2}$ Doctoral Research Fellow at Dpt. of Industrial Engineering, University of Salerno, Fisciano, Salerno, Italy \\ ${ }^{3}$ CEO at eProlnn S.r.l., Fisciano, Salerno, Italy
}

\begin{abstract}
There is a strongly increasing diffusion of Electric Vehicles (EV) and Plug-in Hybrid Electric Vehicles (PHEV), in order to reduce air pollution in urban environment and to mitigate the global warming issues. Anyway, the achievement of this latter goal strictly depends on the source of primary energy used to generate electrical energy. In the paper, a model for the optimal design and operation of a charging station for EV and PHEV assisted by a PhotoVoltaic (PV) plant is presented. A provisional model for the estimation of the incoming insolation, based on cloudiness prevision, is integrated with a nonlinear constrained optimization algorithm, in order to satisfy the load while minimizing the recourse to electrical grid for battery storage charging. Simulations on different locations and charging loads for various size of PV plant and battery capacity are presented, and the benefits in terms of $\mathrm{CO} 2$ reduction discussed.
\end{abstract}

Keywords: Solar, Photovoltaic, Recharge, Model Predictive Control (MPC).

\section{INTRODUCTION}

The growth of world energy consumption and the increase of passenger vehicles are setting new challenges to environmental protection. Global warming mitigation is a pressing issue, and governments and institutions are defining new stringent limits for $\mathrm{CO} 2$ emissions [1].

Large diffusion of Electric Vehicles (EV) and Hybrid Electric Vehicles (HEV) seems to be the most feasible solution to these problems [2]. However, the need of fast charging infrastructure and the still low penetration of renewable electricity production limit the feasibility of a rapid electrification of the fleet. Moreover, a very large reconversion of fleets with massive scrapping of cars often still in good conditions is not the most sustainable solution in a Life Cycle Assessment (LCA) perspective [3], and other options, as ecological car reconversion, are being considered [4].

One of the most critical aspects toward the mitigation of global warming issues by means of fleet electrification is the source of primary energy used to generate electrical energy for recharging the vehicles. It is therefore mandatory to maximize and optimize the recourse to renewable sources, as wind and particularly PhotoVoltaics (PV), to reduce the $\mathrm{CO} 2$

*Address correspondence to this author at the Dpt. of Industrial Engineering, University of Salerno, Fisciano, Salerno, Italy;

E-mail; grizzo@unisa.it production associated to road transport, also with direct use of PV on cars [5].

The problem of designing and managing of recharging stations for electric and plug-in vehicles has attracted many researchers in last decade (Figure 1). Some recent reviews of these studies can be found in [6-8]. Part of these papers, about $10 \%$ of them, has focused on recharging stations assisted by photovoltaic (Figure 2) [9].

In the following, a large but not comprehensive overview of the papers published on such subjects is presented. Besides prevailing use of batteries as storage systems, the recourse to Compressed Air Energy Storage (CAES) [11] and to hydrogen and electrolyzers has been also considered, with the recourse to complex stochastic methodologies [12]. Demand response approach is often used, also considering multi-objective approach to achieve the best compromise between minimizing cost of electricity and polluting emissions [13]. The comprehensive benefits of solar assisted EV charging facilities in a complex urban environment has been also analysed [14]. The dynamic EV charging via the real time coordination between the PV power station, the grid and the storage for enhancing the station working as a stand-alone system as long as possible and satisfying the EVs requirement has been proposed [15]. The time-of-use adjustment method is adopted also by other authors [16], by the charging/discharging priorities calculation and electricity prices. The 


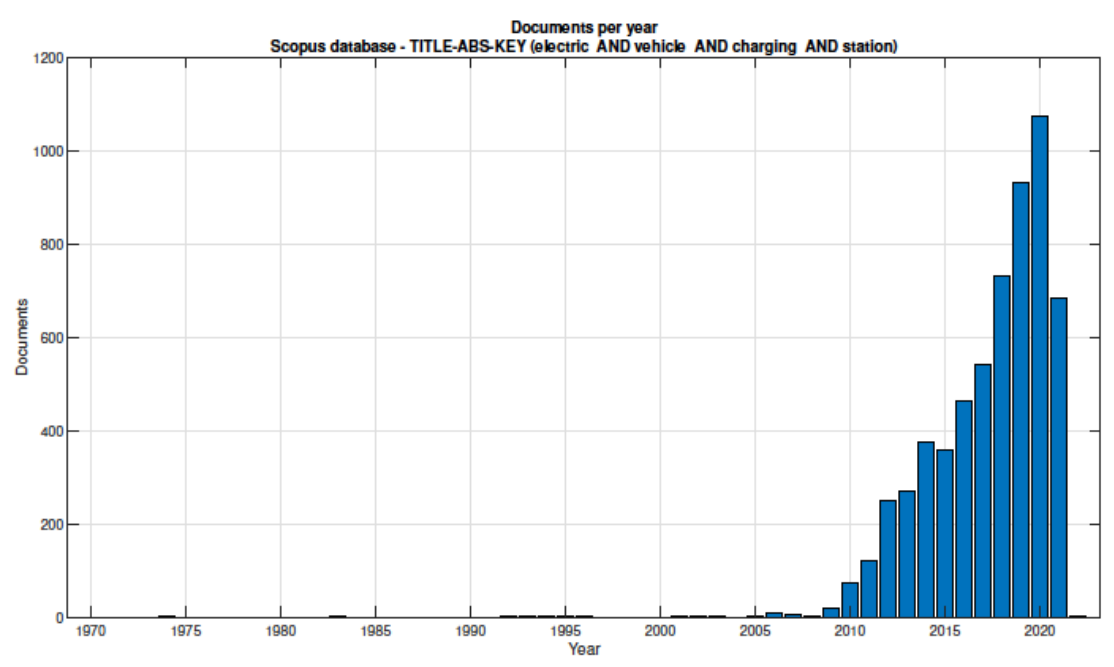

Figure 1: References on Scopus database - TITLE-ABS-KEY (electric AND vehicle AND charging AND station) [10].

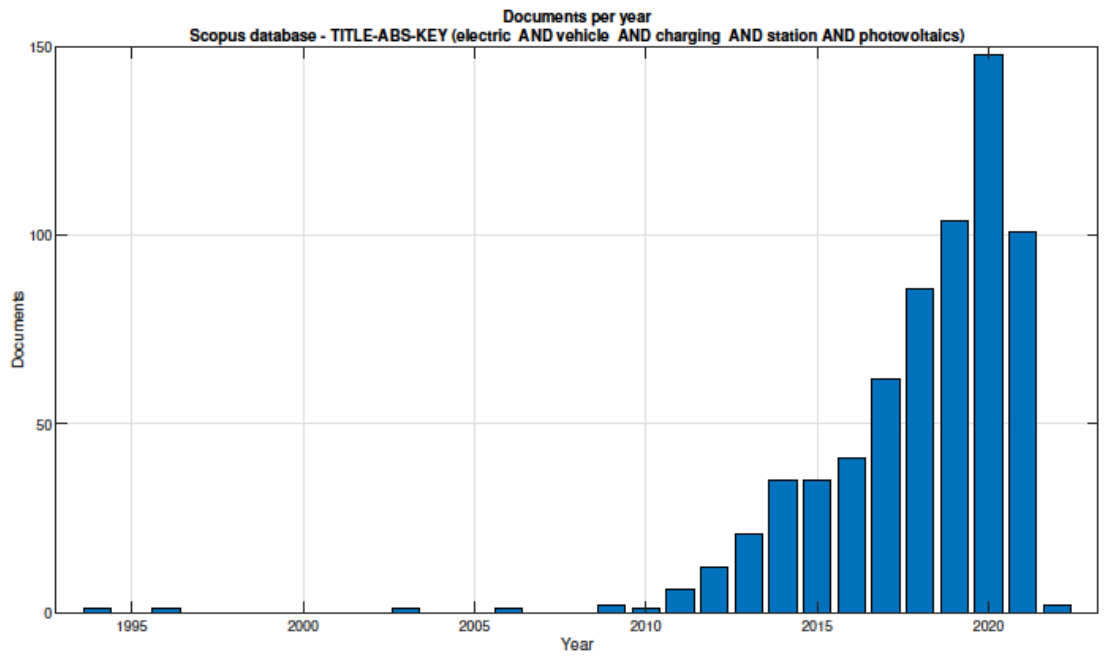

Figure 2: References on Scopus database - TITLE-ABS-KEY (electric AND vehicle AND charging AND station AND photovoltaics) [10].

proposed approach would ensure that the energy usage does not exceed contract capacity, giving also directions for optimizing the contract capacity. A mathematical optimization approach for the optimal configuration of a grid-connected electric vehicle extreme fast charging station considering integration of photovoltaic and energy storage has been also studied [17]. The proposed model minimizes the annualized net cost, including investment and maintenance cost of charging ports, PV and energy storage, net cost of purchasing energy from utility and selling energy to EV customers, degradation cost of energy storage and demand charge. In the paper [17] a day-ahead optimal scheduling model via Mixed-Integer Linear Programming (MILP) is proposed. Constraints include the active power balance between load demands and generation, the maximum/minimum output power of $P V$, wind turbine and diesel generation, the maximum charging power and the charging time.

Most of the papers have focused their analysis on the methodologies for optimal management of such plants, considering different approaches to estimate the vehicle scheduling. In spite of a large attention to a such fundamental problem, a relatively little effort has been spent on considering the benefits associated to a prediction of solar irradiation. Indeed, the theoretical solar irradiation in sunny conditions can be computed with high precision [18], while the prediction of the cloudiness index can be now available for many locations by on-line services [19], so allowing to estimate the real irradiation over a time horizon of several hours. 
In this paper, the models used to predict solar irradiance taking into account the cloudiness index are reviewed [18]. Then, a mathematical model for the optimal design and operation of a charging station for EV and PHEV assisted by a PV plant is presented. The model of the recharging station is integrated with a nonlinear constrained optimization algorithm, in order to satisfy the incoming load while minimizing the recourse to electrical grid for battery storage charging. Simulations on different locations and charging loads for various size of PV plant and battery capacity are presented, and the benefits in terms of cost and $\mathrm{CO} 2$ reduction discussed. A specific goal of the paper is assessing the benefits achievable by the knowledge of the incoming solar irradiance in a given time horizon (typically 48 hours), in order to optimize the operation of the battery and minimize the recourse to the grid. To this end, the results obtained with a predictive model are compared with those achieved by using a heuristic non-predictive approach.

\section{PREDICTION OF SOLAR IRRADIANCE}

In the following, some models proposed in literature and validated by [18] are reviewed: the modified BCLS model, the Kasten model and the Munro model. In order not to affect the fluency of this work, an overview of the astronomical and general formulas are given in Appendix A.

\subsection{The Modified BCLS Model}

In cloudless sky condition, the direct beam $I_{0}$ $\left[\mathrm{W} / \mathrm{m}^{2}\right]$ and diffuse irradiance $D_{0}\left[\mathrm{~W} / \mathrm{m}^{2}\right]$ on a horizontal surface are given by:

$$
\begin{aligned}
I_{0} \quad=G_{0}\left(a_{1}+b_{1} u_{w}-a_{3}(d-400)\right) \exp \left(-\left(a_{2}+b_{2} u_{w}\right.\right. \\
\\
\left.\left.\quad+b_{3}(d-400)\right) m\right) \\
D_{0}=k\left(I_{w}-I_{0}\right) \\
a_{1}=-0.13491, a_{2}=0.13708, a_{3}=3.68 \cdot 10^{-5} \\
b_{1}=-4.28 \cdot 10^{-3}, b_{2}=2.61 \cdot 10^{-3}, b_{3}=1.131 \cdot 10^{-4}
\end{aligned}
$$

where $d$ is the dust content (200 particles per $\mathrm{cm} 3$ ), $k$ is an empirical coefficient equal to $k=0.5\left(\cos \vartheta_{z}\right)^{\frac{1}{3}}$ and $I_{w}\left[\mathrm{~W} / \mathrm{m}^{2}\right]$ is the direct irradiance transmitted in absence of scattering, given by:

$$
\begin{aligned}
I_{w} & =G_{0}(0.938 \exp (-0.00154 X)) \\
& +\left(2.97 X^{2.1}-773.24 \cdot 10^{-5} X^{3}+85058.73 \frac{1+X}{1+10 X^{2}}\right) 10^{-3}
\end{aligned}
$$$$
\text { with } X=m u_{w}
$$

If a relative sunshine $S$ computed as $S=(1-P C)$ where $P C$ is the cloudiness, the direct and diffuse irradiance in cloudy sky are given by:

$$
\begin{aligned}
& I \quad=S I_{0} \\
& D=S D_{0}+t(1-S)\left(I_{0}+D_{0}\right)
\end{aligned}
$$

where $t$ is the cloud transmissivity factor which can be calculated as:

$t=0.61-0.015 L A T+0.0002 L A T^{2}$

Equation (4) has been verified for latitudes from 40 to 50 degrees.

The global irradiance $G\left[W / \mathrm{m}^{2}\right]$ is given by:

$$
G=\frac{I+D}{1-a(0.2+0.5(1-S))}
$$

where $a$ is the albedo factor ( $a=0.15$ for asphalt ground)

Authors of this study propose a slight modification of this model, in order to consider a tilted surface. In this case, Equation (3) can be modified considering Equations (36) and (37) to include the view factors and the reflected irradiance $R$ :

$$
\begin{array}{ll}
I^{*} & =S I_{0} R B \\
D^{*} & =F_{s k y}\left(S D_{0}+t(1-S)\left(I_{0}+D_{0}\right)\right) \\
R^{*} & =F_{\text {gro }} a(I+D)
\end{array}
$$

Consequently, Equation (5) becomes:

$$
G=\frac{I^{*}+D^{*}+R^{*}}{1-a(0.2+0.5(1-S))}
$$

\subsection{The Modified Kasten Model}

The global irradiance $G\left[\mathrm{~W} / \mathrm{m}^{2}\right]$ for a cloudy sky on a horizontal surface is given by:

$$
G=G_{0}\left(1-0.72 P C^{3.2}\right)
$$

where $G_{0}\left[\mathrm{~W} / \mathrm{m}^{2}\right]$ is the global irradiance for cloudless sky evaluated with the MAC model:

$$
G_{0}=I_{0}+D_{r}+D_{a}
$$

where $I_{0}\left[\mathrm{~W} / \mathrm{m}^{2}\right]$ is the direct component of the irradiance and $D_{r}$ and $D_{a}\left[\mathrm{~W} / \mathrm{m}^{2}\right]$ are the diffuse components due to Rayleigh and aerosol scattering, respectively: 


$$
\begin{aligned}
& I_{0}=G_{0}\left(\tau_{0} \tau_{r}-a_{w}\right) \tau_{a} \\
& D_{r}=G_{0} \tau_{0} \frac{1-\tau_{r}}{2} \\
& D_{a}=G_{0}\left(\tau_{0} \tau_{r}-a_{w}\right)\left(1-\tau_{a}\right) a_{a} g
\end{aligned}
$$

where $G_{0}\left[\mathrm{~W} / \mathrm{m}^{2}\right]$ is the extraterrestrial irradiance on a horizontal surface, $\tau_{0}, \quad \tau_{r}$ and $\tau_{a}$ are the transmissivities for absorption by ozone, for Rayleigh scattering and for extinction by aerosol, respectively, $a_{w}$ is the absorptivity by water vapor, $a_{a}$ is the spectrally-averaged single-scattering albedo for aerosols, while $g$ is the ratio of forward to total scattering by aerosols.

The transmissivity for absorption by ozone $\tau_{0}$ of an assumed ozone layer $u_{0}$ with $3.5 \mathrm{~mm}$ of thickness is computed by:

$$
\begin{aligned}
x_{0} & =u_{0} m \\
a_{0} & =\frac{0.1082 x_{0}}{1+13.86 x_{0}^{0.805}}+\frac{0.00658 x_{0}}{1+\left(10.36 x_{0}\right)^{3}} \\
& +\frac{0.00218}{1+0.0042 x_{0}+0.00000323 x_{0}^{2}} \\
\tau_{0} & =1-a_{0}
\end{aligned}
$$

The transmissivity for Rayleigh scattering $\tau_{r}$ is given by:

$$
\begin{aligned}
\tau_{r} & =0.9768-0.0874 m+0.010607552 m^{2}-8.46205 \cdot 10^{-4} m^{3} \\
& +3.57246 \cdot 10^{-5} m^{4}-6.0176 \cdot 10^{-7} m^{5}
\end{aligned}
$$

The transmissivity for extinction by aerosol $\tau_{a}$ is given by:

$\tau_{a}=k_{a} m$

where the unit air mass aerosol transmissivity, $k_{a}$, is equal to 0.90 .

The absorption by water vapor is determined as:

$$
\begin{aligned}
a_{w} & =\frac{0.29 x_{2}}{\left(1+14.15 x_{2}\right)^{0.635}+0.5925 x_{2}} \\
& \text { with } x_{2}=m u_{w}
\end{aligned}
$$

Lastly, the spectrally-averaged single-scattering albedo for aerosols $a_{a}$ is equal to 0.75 and the ratio of forward to total scattering by aerosols, $g$, is given by:

$g=0.93-2.21 \ln m$

Analogously to the modified BCLS model, in order to consider a tilted surface, Equation (10) should be modified as follows:

$$
\begin{aligned}
I_{0}^{*} & =G_{0}\left(\tau_{0} \tau_{r}-a_{w}\right) \tau_{a} \\
D^{*} & =\left(D_{r}+D_{a}\right) F_{s k y} \\
R^{*} & =a\left(\frac{I_{0}}{R B}+D_{r}+D_{a}\right) F_{g r o}
\end{aligned}
$$

\subsection{The Munro Model}

The direct irradiance under cloudy sky, I $\left[\mathrm{W} / \mathrm{m}^{2}\right]$, on a horizontal surface is given by:

$I=I_{0}(1-P C)$

where $I_{0}$ is the direct irradiance under clear sky calculated as for eq:Kasten_direct_diffuse.

The diffuse irradiance under cloudy sky, $D\left[\mathrm{~W} / \mathrm{m}^{2}\right]$, is given by:

$$
D=D_{1}+D_{2}
$$

where $D_{1}$ and $D_{2}$ correspond to the diffuse radiation from the cloudless portion of the sky plus the radiation that passes through the cloud layer and the diffuse radiation due to the reflections between cloud and ground. They are determined via the following equations:

$$
\begin{aligned}
& D_{1}=D_{0}(1-P C)+P C I_{0}\left(1-a_{n}-\alpha_{n}\right) \\
& D_{2}=\frac{\left(I+D_{1}\right) P C \alpha_{b} a}{1-\alpha_{b} a}
\end{aligned}
$$

where $D_{0}$ is the cloudless sky diffuse radiation, $a_{n}$ is the absorptance of the cloud, $\alpha_{n}, \alpha_{b}$ and $a$ are the reflectance of the cloud top, the cloud base and the albedo of the ground, respectively. $D_{0}$ is evaluated as the sum of $D_{r}$ and $D_{a}$ from eq:Kasten_direct_diffuse, respectively. Common values are: $a_{n}=0.18, \alpha_{b}=0.6$ and $a=0.2 . \alpha_{n}$ is calculated as:

$$
\begin{aligned}
\alpha_{n} & =1+\left(\exp (-0.5656 m x)\left(x(1-1.48 m)+3.58 m^{-1}-2.62\right)\right. \\
& \left.-3.54 m^{-1}-2.62\right)(x+5.2)^{-1}
\end{aligned}
$$

where $x$ is the ratio of cloud thickness to the mean free path of light through the cloud. $x$ was set equal to 1.5.

The global irradiance on cloudy sky $G$ on a horizontal surface is given by:

$G=I+D$

When a tilted surface is considered, from Equation (19) it is possible to include view factors and reflected irradiance as follows: 


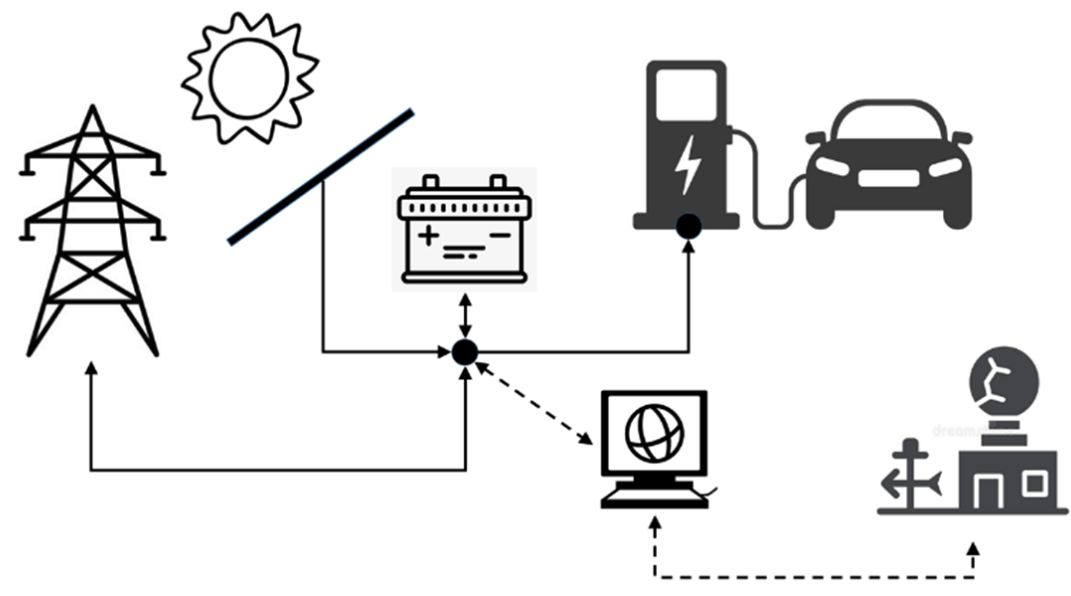

Figure 3: Scheme of the recharging station.

$$
\begin{aligned}
& D^{*}=\left(D_{1}+D_{2}\right) F_{s k y} \\
& R^{*}=\left(D_{1}+D_{2}\right) F_{g r o}
\end{aligned}
$$

\section{METHODOLOGY}

In order to assess the benefits achievable with the prediction of solar irradiation in the energy management of a recharging station assisted by photovoltaics for plug-in vehicles, a mathematical model of the system has been developed in Matlab. The scheme of the plant is presented in Figure 3.

The electric energy flowing from the PV panels, from/to the battery and from/to the grid concur to recharge the cars, whose hourly load is considered known. A computer is connected via Internet to a provider of meteo data, achieving the prediction of cloudiness index for the place where the recharging station is located.

A possible scheme of architecture for real time Model Predictive Control (MPC) is presented in Figure 4. A submodel provides the updated estimation of the electrical load for vehicle recharging based on historical data and external information on traffic and other exogenous variables. Another submodel estimate the incoming solar power in the next time horizon (i.e. 48 hours), combining the prediction of cloudiness index with solar radiation models. These data, together with the battery state of charge and the actual electric load, are supplied to the plant model, integrated with an optimization algorithm. The output is the optimal power flow from/to the battery and the grid in the electrical node.

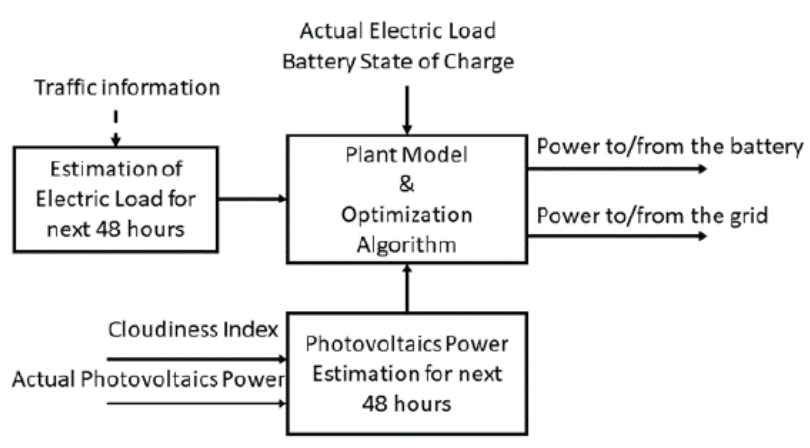

Figure 4: Scheme of the real-time control of the plant.

\subsection{Predictive Optimal Model}

A model of solar irradiation then predicts the net solar power for next 48 hours, and a model of the plant integrated with a non-linear constrained optimization algorithm determines the optimal strategy for exchanging electric power with the grid, in order to satisfy the load (i.e. recharging the vehicles) while minimizing the cost of the energy (or, in alternative, the impact on $\mathrm{CO} 2$ emissions):

$\min _{X} f(X)=\sum_{i=1}^{n} P_{G, \max } \Delta h C_{i}$

where $P_{G, \max }$ is the maximum power that can be exchanged with the grid (drawn or delivered). The decision variables $X_{i}$ represent the fraction of the maximum power exchanged with the grid. They can therefore vary from -1 (maximum power delivered to the grid) to 1 (maximum power purchased from the grid). The time horizon is equal to 48 hours $(n=48)$, and the time interval $\Delta h$ is assumed equal to 1 hour. 
Table 1: Cost of the Electric Energy

\begin{tabular}{|c|c|c|}
\hline Case & $\begin{array}{c}\text { Cost of the Energy } \\
€ / \text { kWh }\end{array}$ & Range \\
\hline \hline F1 & 0.07516 & $X_{i}>0, h=8-19$ \\
\hline F2 & 0.06148 & $X_{i}>0, h=0-8,19-24$ \\
\hline F0 (no time slots) & 0.06595 & $X_{i}>0, h=0-24$ \\
\hline CV (sale to the grid) & 0.03980 & $X_{i}<0, h=0-24$ \\
\hline CV (no sale to the grid) & 0 & $X_{i}<0, h=0-24$ \\
\hline
\end{tabular}

The unit energy cost $C_{i}$ is assigned as a function of the day hour when drawing energy from the grid $\left(X_{i}>0\right)$, if time slots are considered (F1 and F2), while it may have a constant value in case the cost is not modulated with the hour (case F0). The possibility to delivering the power to the grid is also considered $\left(X_{i}<0\right)$, with a price, $C V$, consistently lower that the cost of purchasing power from the grid (F0, F1 and F2). The possibility that energy cannot be sold to the grid (i.e. $C V=0$ ) has been also considered.

In the computations, the data presented in Table 1, which are consistent with the Italian market, are assumed.

The power entering of leaving the battery, $P_{B}$, is then determined by the values of load, $P_{L}$, and by the power from PV panels, $P_{P V}$. When energy is stored in the battery $\left(P_{B}>0\right)$, a Coulombic efficiency $\eta_{B}$ is considered to take into account Joule losses associated to the current (it is assumed constant and equal to 0.95 ). A value 1 is assumed when energy is drawn from battery:

$P_{B}=\left(P_{P V}+X_{i} P_{G, \max }-P_{L}\right) \eta_{B}$
The State of Charge (SOC) in then determined as a function of the battery capacity, $C_{B}$, starting from the initial value $S O C_{1}$, considered known:

$S O C_{i}=S O C_{i-1}+P_{B} \frac{\Delta h}{C_{B}}$

An equality constraint on the final value of the State of Charge is imposed (terminal constraint) as well as further inequality constraints expressing the condition that the State of Charge must stay between 0 and 1, at any time:

$$
\begin{aligned}
& \operatorname{SOC}_{n}=\operatorname{SOC}_{1} \\
& \operatorname{SOC}_{i} \geq 0, \operatorname{SOC}_{i} \leq 1, i=1, n
\end{aligned}
$$

\subsection{Heuristic Rule Based Model}

In order to assess the benefits of the algorithm based on predictive model, a second method based on heuristic approach has been considered. The energy management strategy is based on the rules listed in

This strategy does not necessarily result in a final value of SOC equal to the initial one, as for the optimal strategy. Therefore, in order to make a correct

Table 2: Rules for Heuristic Strategy

\begin{tabular}{|c|l|}
\hline Case & \\
\hline \hline$P_{P V} \geq P_{L}$ & $\begin{array}{l}\text { The load is satisfied by photovoltaics, and the extra power is sent to the battery; } \\
\text { if the extra power exceeds the maximum battery capacity, the difference is delivered to the grid; } \\
\text { if the power to be delivered to the grid is greater than maximum power, the difference is lost. }\end{array}$ \\
\hline $\begin{array}{c}P_{P V}<P_{L} \\
S O C \geq S O C_{n}\end{array}$ & $\begin{array}{l}\text { The power from photovoltaic is used to partly satisfy the load; } \\
\text { the missing power is drawn from the battery; } \\
\text { no power is taken from the grid. }\end{array}$ \\
\hline $\begin{array}{c}P_{P V}<P_{L} \\
S O C<S O C_{n}\end{array}$ & $\begin{array}{l}\text { The power from photovoltaic is used to partly satisfy the load; } \\
\text { the missing power is partly drawn from the battery and partly taken from the grid. }\end{array}$ \\
\hline
\end{tabular}


comparison between the two strategies the cost corresponding to the missing or extra energy in the battery at the final stage $(i=n)$ is accounted by the following formula:

Cost $=\sum_{i=1}^{n} X_{i} P_{G, \max } \Delta h C_{i}+\left(S O C_{1}-S O C_{n}\right) C_{B} k$

where the unit cost $k$ is equal to F0 in case of purchasing and equal to $C V$ in case of delivering the energy to the grid.

\subsection{Load Profiles}

Three different profiles, shown in Figure 5, for load have been considered, with a total charge of $69 \mathrm{kWh}$ within the day (and $138 \mathrm{kWh}$ in the time horizon). The profile 1 has a prevailing recharge during the day, the profile 2 takes most of the energy during the night, and profile 3 has a constant load in the 24 hours.

\section{RESULTS}

In order to assess the effect of the different conditions and variables on the benefits achievable by the recourse to a Model Predictive Control approach, a systematic comparison with the cost and $\mathrm{CO} 2$ obtained with the two strategies has been performed. The conditions analyzed are described in Table 3.
Table 3: Conditions Analyzed in the Study

\begin{tabular}{|l|c|c|}
\hline \multicolumn{2}{|c|}{ Description } & Values \\
\hline \hline PV Area & $\mathrm{m} 2$ & 0 - 100 \\
\hline Tilt angles & $\mathrm{deg}$ & 20 \\
\hline Azimuth & $\mathrm{deg}$ & 0 (South) \\
\hline PV efficiency & - & 0.18 \\
\hline Days of the year & - & January 1st - July 1st \\
\hline Time horizon & $\mathrm{h}$ & 48 \\
\hline Battery capacity & $\mathrm{kWh}$ & $1-30$ \\
\hline Load profiles & - & $1-2$ - 3 \\
\hline Control strategy & - & A-Predictive, B-Heuristic \\
\hline CO2 emission factor (grid) & $\mathrm{gCO}_{2}$, eq/kWh & 397 \\
\hline
\end{tabular}

The emission factor for the electric energy provided to the recharge of electric vehicles is set to 397 $\mathrm{gCO} 2, \mathrm{eq} / \mathrm{kWh}$, as determined by the Italian regulations [6]. The power coming from PV panels is considered carbon free.

Figure 6 present the solar data in the provisional horizon of 48 hours for two representative conditions (January 1st and July 1st), for the assigned location. The global irradiance is estimated starting from direct theoretical irradiance and cloudiness index. Solar height, solar azimuth and incidence angle are also plotted, in the two cases.
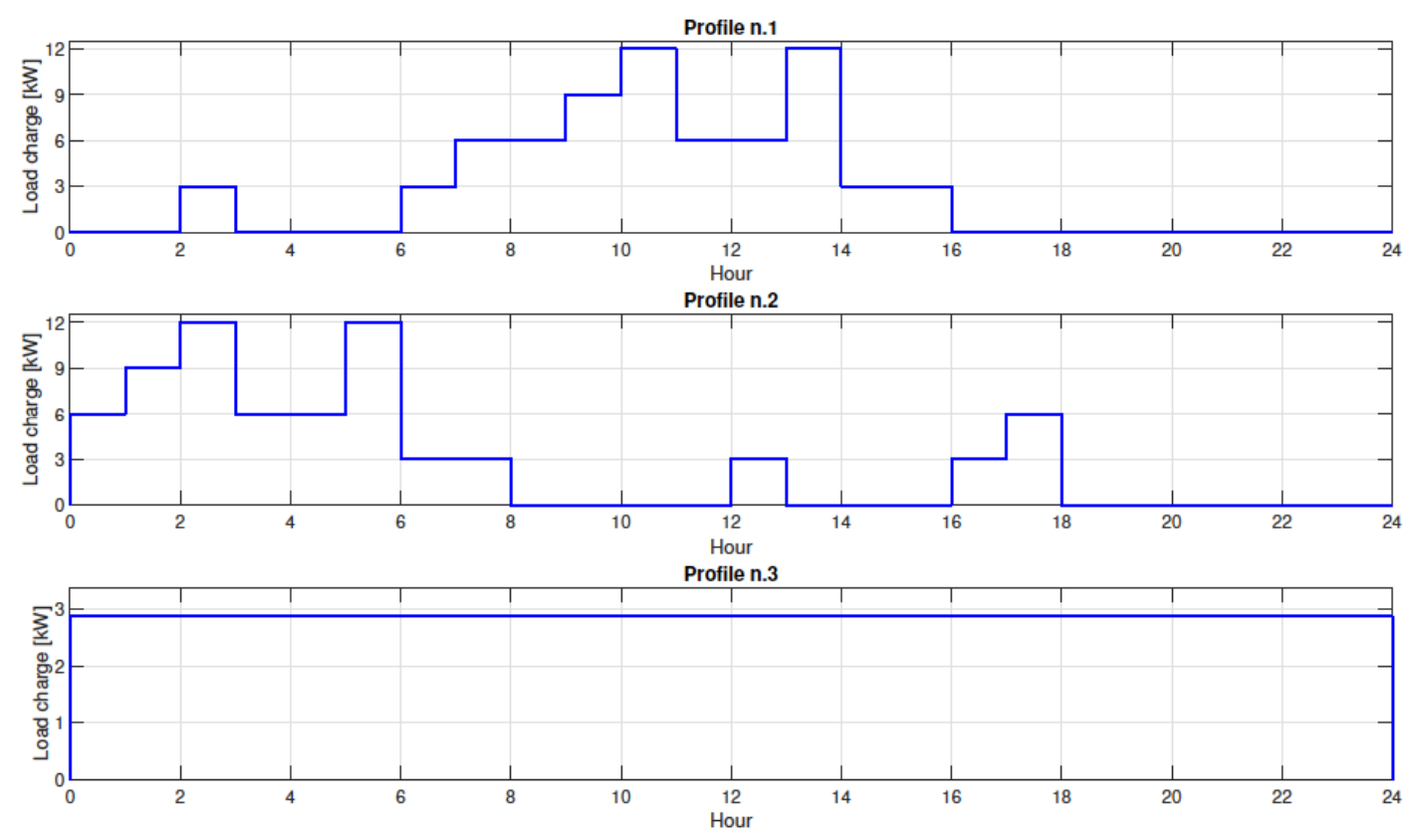

Figure 5: Load profiles for vehicle recharging. 

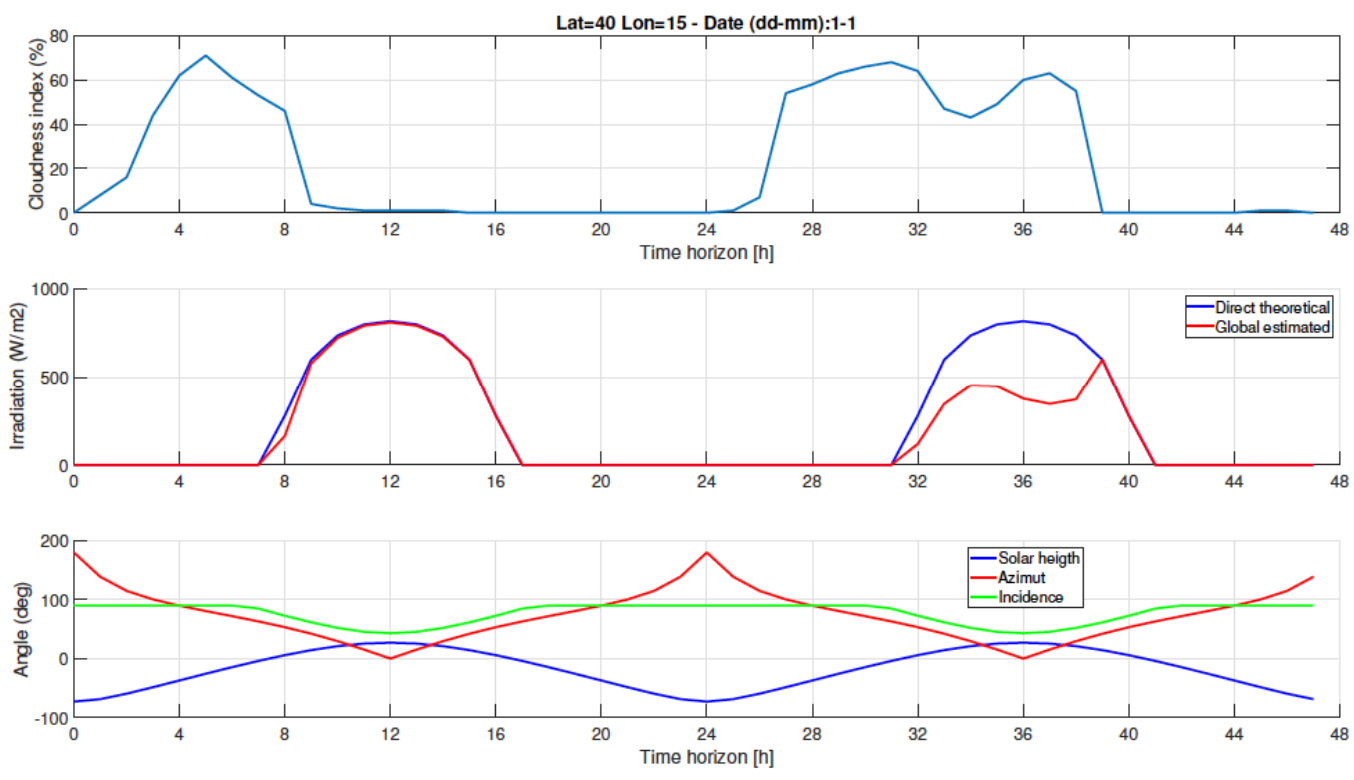

Figure 6: Solar irradiance, cloudiness, solar height, azimuth and incidence angles (January 1st).

Figures 8 and 9 present an example of the results of simulations for provisional optimal strategy and heuristic strategy, respectively. The conditions are:

- Battery capacity: $5 \mathrm{kWh}$,

- Day: July 1st,

- Area of PV panels: $30 \mathrm{~m} 2$,

- Load profile: 1 (Figure 5)

In both cases, PV panels have provided $67 \mathrm{kWh}$ and the load (138 kWh) has been satisfied. Is case of provisional strategy, $71.2 \mathrm{kWh}$ have been drawn from the grid, with a cost of $5.22 €(0.0379 \in / \mathrm{kWh})$, while in case of heuristic strategy $82.9 \mathrm{kWh}$ have been drawn, with a cost of $6.05 €(0.0438 € / \mathrm{kWh})$. In this case, the recourse to provisional model allows to save $13.6 \%$ of cost. It can be also noticed that in case of predictive strategy the final values of State of Charge $(0.7)$ is equal to the initial one (Figure 5), while this is not true for the heuristic strategy, where the final SOC is 0.57 , and an additional amount of electrical energy $(0.66 \mathrm{kWh})$ is drawn from the grid to make a fair comparison with the case of provisional strategy.

The benefits of the provisional strategy with respect to the heuristic one is due to different mechanisms: i) avoiding/limiting the lost of extra power from PV panels due to unavailability of battery capacity; this occurrence can be avoided thanks to the prediction of the incoming solar power and to the adoption of proper strategies to keep adequate capacity in the battery; ii) lower recourse to power delivering to the grid, whose price is consistently lower than the cost paid for buying energy from the grid; also this goal can be achieved thanks to the prediction capabilities and to the optimization algorithm, with the adoption of strategies that keep free capacity in the battery; iii) optimizing the purchase of power from the grid, privileging the time slots with lower cost; iv) limiting the excursions of battery SOC, associated with Coulombic losses; this results is achieved thanks to the constraint on the final SOC value in the constrained optimization algorithm, that naturally limits the SOC excursions.

Graphs shown in Figure 10 (cases: C01 and C02) present the case of simulations in winter while graphs shown in Figure 10 (cases: $\mathrm{C} 03$ and C04) present those in summer, for different area of PV panels (from 0 to $100 \mathrm{~m} 2$ ). In these cases, a quite large battery capacity has been considered ( $30 \mathrm{kWh}$ ). Two scenarios has been analyzed: when it is possible to sale extra power to the grid (cases: $\mathrm{C} 01$ and C03), or not (cases: C02 and C04).

As expected, the cost (in $€ / \mathrm{kWh}$, normalized to the load) decreases when increasing the total energy harvested from PV panels. In summer time, the PV power can completely satisfy the load (i.e. cost is equal to zero), and also reach negative values when extra power is sold to the grid (case C03). When the area pf $\mathrm{PV}$ is zero there is no differences between the four cases, since all the energy is drawn from the grid. The average cost with predictive strategy is lower than the heuristic one, since the optimal strategy tend to reduce 

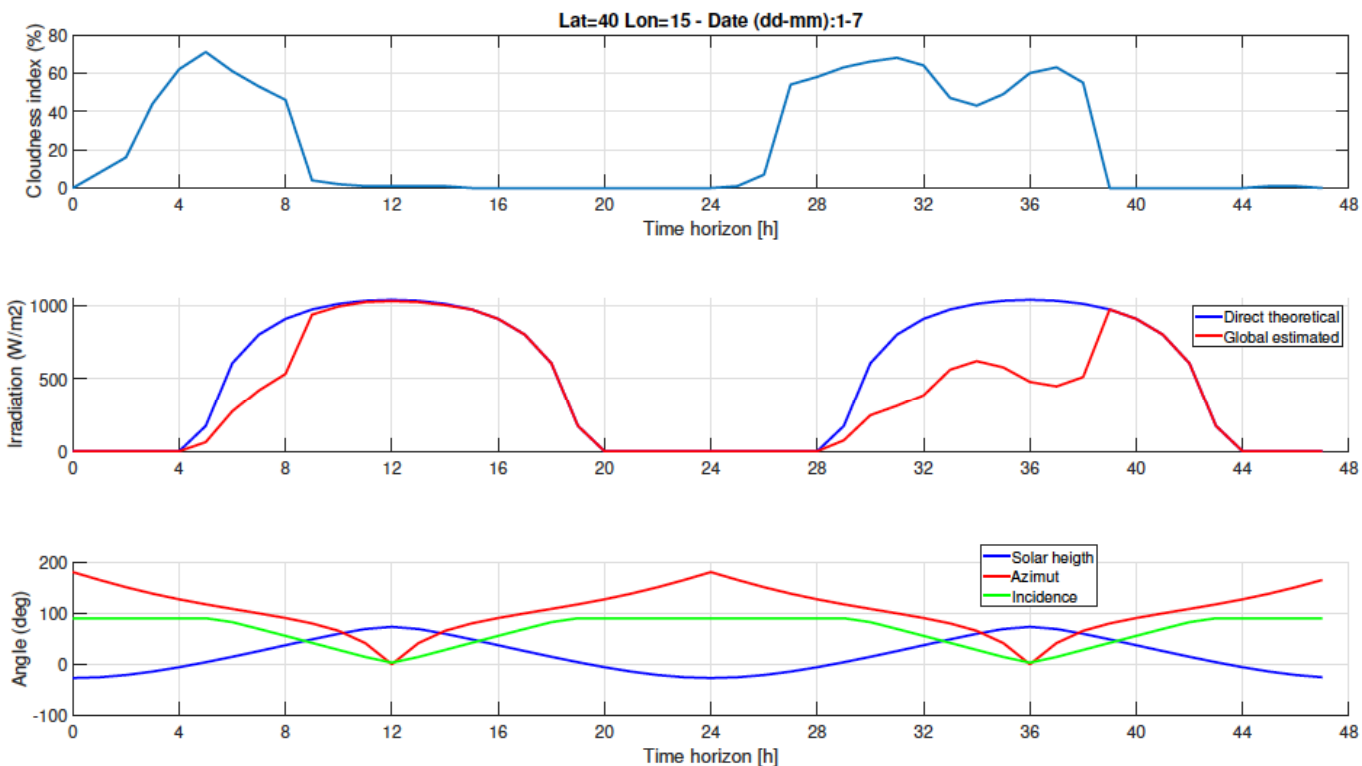

Figure 7: Solar irradiance, cloudiness, solar height, azimuth and incidence angles (July 1st).

the power purchasing in the hours when the cost is higher. It can be also observed that there are no differences between the results in the cases shown in Figure 10 (cases: C01 and C02): in these cases, in winter time there is no extra power to sale to the grid.

In all the examined cases, the cost achieved with the provisional strategy is lower than the one obtained with the heuristic strategy. The relative gain increases when the PV contribute is higher.

\subsection{Effect of Battery Capacity and of Load Profile}

The study has been extended by varying battery capacity, and for different load profiles (Figure 5). For each series of simulations, PV area has been varied from 0 to $100 \mathrm{~m} 2$ (as in cases shown in Figure 10). Each series of results has been characterized by an average gain of the predictive strategy in terms of energy cost $(€ / \mathrm{kWh})$ and $\mathrm{CO} 2$ emissions. The results are presented in Figure $\mathbf{1 1}(a-b-c)$. To add generality to
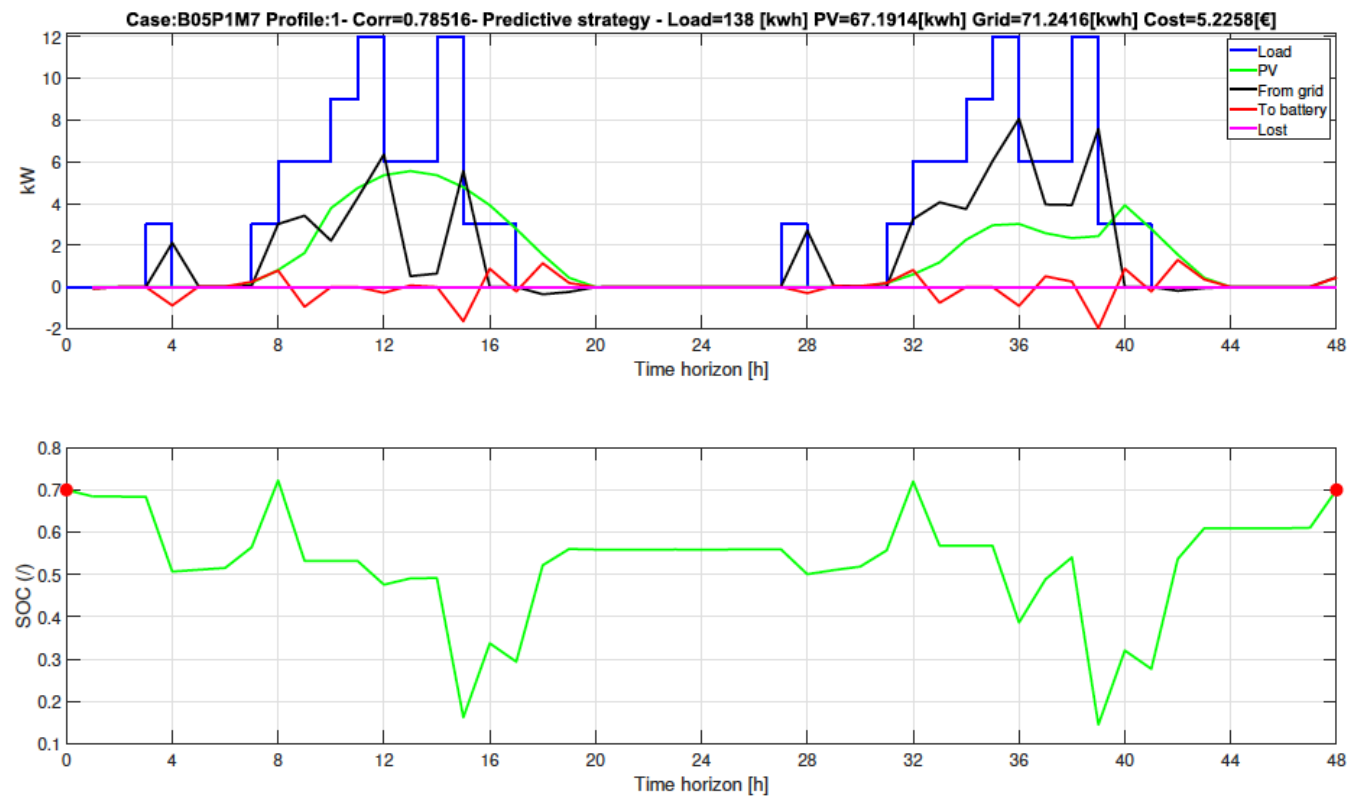

Figure 8: Simulation with predictive strategy (July 1st). 

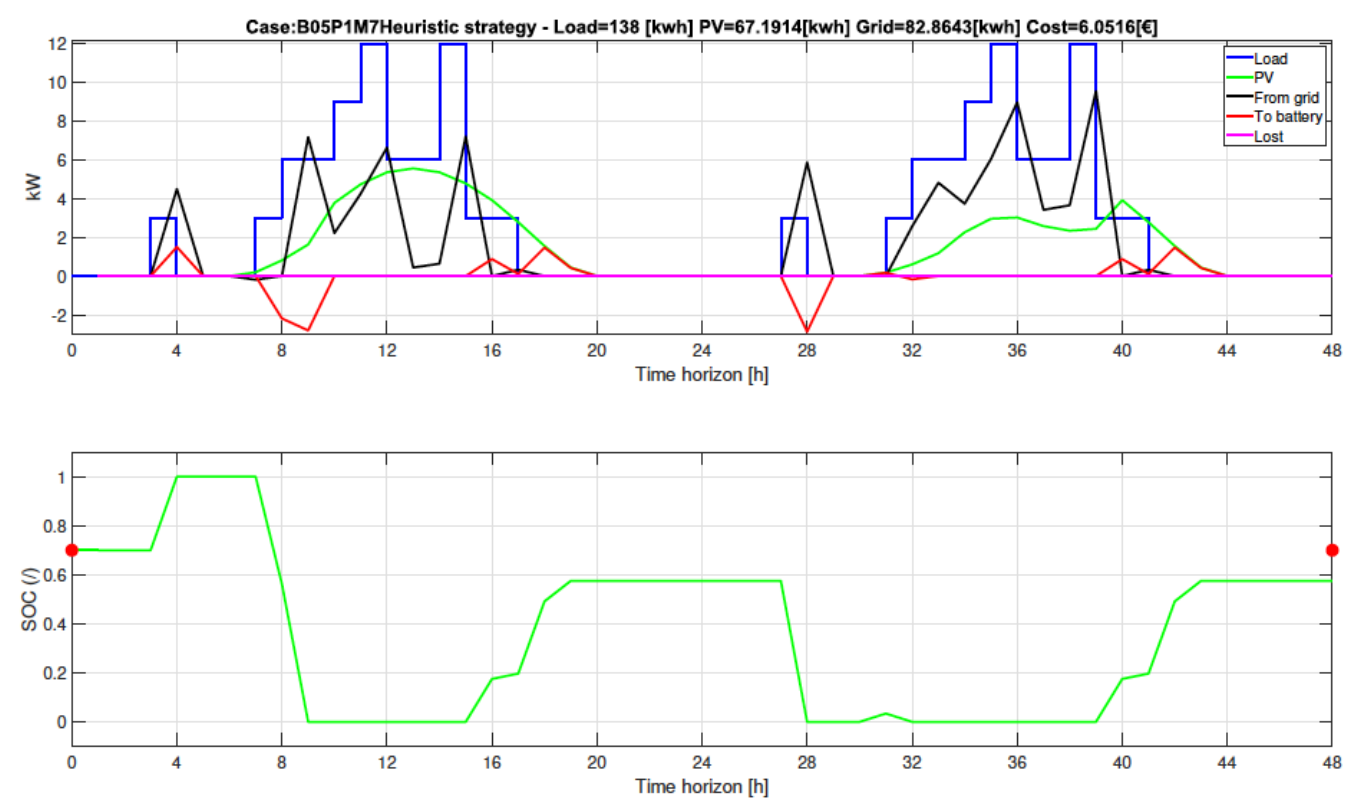

Figure 9: Simulation with heuristic strategy (July 1st).

the results, the battery capacity has been expressed in percent with respect to the day load (69 kWh).

The results show that the percent gain obtainable by the use of a predictive model with respect to the given heuristic strategy in terms of reduction of energy cost is in many cases between $20 \%$ and $30 \%$. Lower values are reached with small battery capacity, since the added value of the algorithm essentially consists in a better use of the battery. Some irregularities can be also noticed (i.e. a negative gain for Profile 2), and some not regular trends in the zone between $5 \%$ and $15 \%$ of percent battery capacity. In all the investigated cases, the best results are obtained starting from about $5 \%$ of capacity battery with respect to daily load. Some presence of irregularities may be also due to presence of local minima in the optimization process, or to incomplete convergence to the results.

The benefits in terms of $\mathrm{CO} 2$ reduction (orange point) have similar trends with respect to the cost reduction (blue points), but attain lower values. This may be explained considering that the objective function of the optimization process is expressed in terms of cost reduction, and not of $\mathrm{CO} 2$ reduction. Moreover, reducing the recourse to grid energy in the period with higher costs (i.e. F1 tariff) and increasing the purchasing in the period of lower cost (i.e F2 tariff) reduces the total cost but does not change the $\mathrm{CO} 2$ impact, that may become even higher. In other words, the two objectives (cost reduction and $\mathrm{CO} 2$ reduction) are strongly correlated but not equivalent. $A$ comparison of results with the three profiles evidences no relevant differences between the cases 1 (prevailing load in the day) and 2 (prevailing load during the night), while the benefits of the predictive strategy for profile 3 (uniform load) seem lower than the other cases, at higher battery capacity. Anyway, further investigations seem necessary to better assess the relationship between PV power, load and battery capacity.

\section{CONCLUSIONS}

In order to maximize the environmental benefits of the electric mobility, an increasing diffusion of recharging stations connected to the electrical grid and powered by photovoltaic panels is expected. The study presented in this paper has shown that the recourse to mathematical models to predict the incoming solar power integrated with tools of mathematical optimization can results in a smarter management of the battery and in a lower and cheaper access to the grid, for three different profiles of load representative of realistic cases. The benefits in terms of cost reduction ranges between $20 \%$ and $30 \%$ in many cases, with respect to non-predictive heuristic strategies. A reduction on $\mathrm{CO} 2$ production between $10 \%$ and $20 \%$ is also achieved. In order to maximize the benefits, further developments seem necessary to integrate the optimal management with the combined prediction of incoming solar power and of the recharging load, based on statistical techniques and on real-time data processing. 


\section{ACKNOWLEDGMENTS}

This study is supported by a grant from European Union (LIFE-SAVE Solar Aided Vehicle Electrification LIFE16 ENV/IT/000442).

\section{APPENDIX: ASTRONOMICAL AND GENERAL FORMULAE}

Solar constant $I_{S C}$, that is the solar electromagnetic radiation per unit area measured at a distance of 1 au from the Sun, is equal to $1367 \mathrm{~W} / \mathrm{m}^{2}$. The intensity of
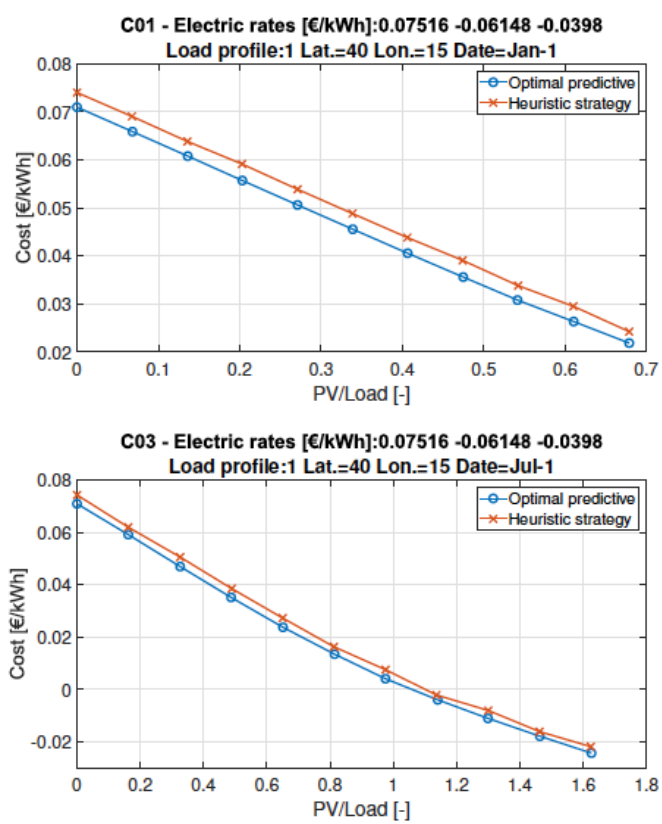

Figure 10: Examined Cases.
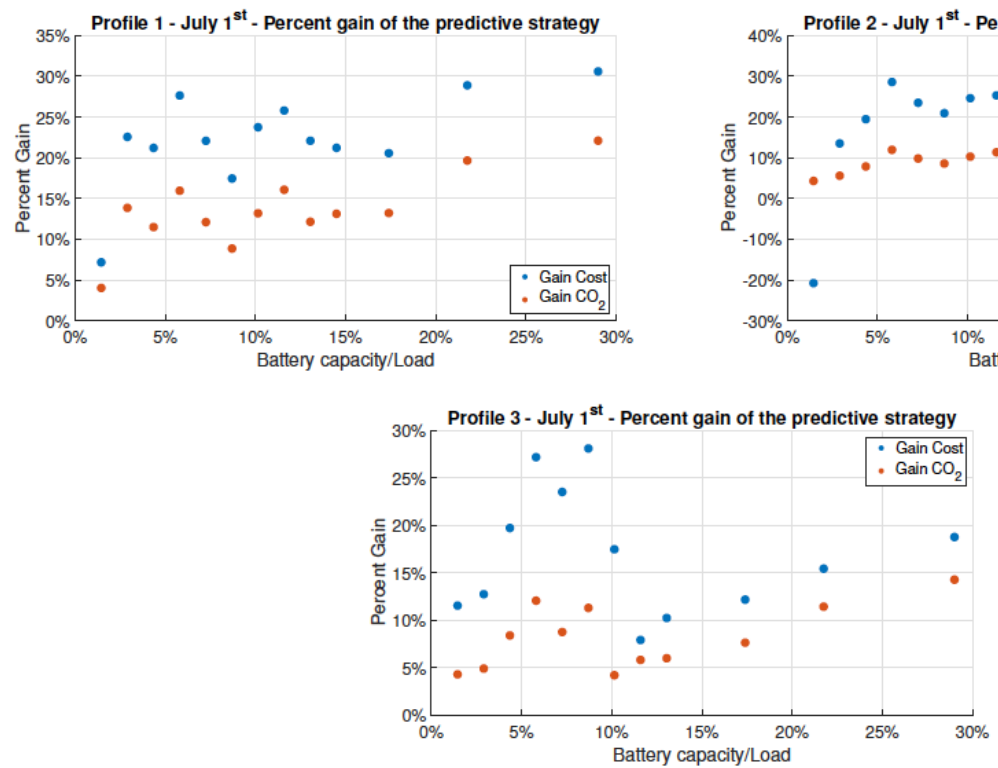

Figure 11: Average gain of predictive strategy with different battery capacity and load profiles.

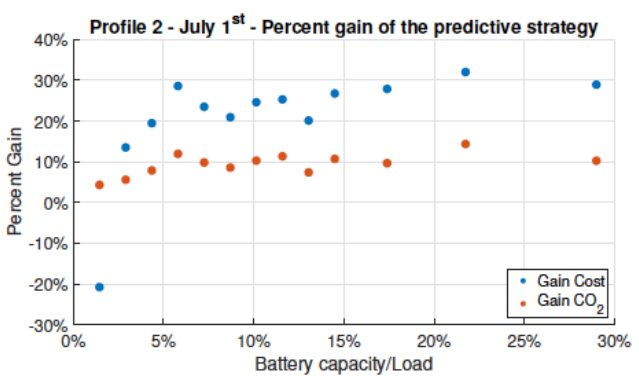

the extra terrestrial solar radiation $I_{0}\left[\mathrm{~W} / \mathrm{m}^{2}\right]$ in a given year day $n$ is computed with:

$I_{0}=I_{S C}\left(1+0.033 \cos \frac{2 \pi n}{365}\right)$

The extra-terrestrial solar irradiance on a horizontal surface $G_{0}\left[\mathrm{~W} / \mathrm{m}^{2}\right]$ is determined by:

$G_{0}=I_{0} \cos \vartheta_{z}$
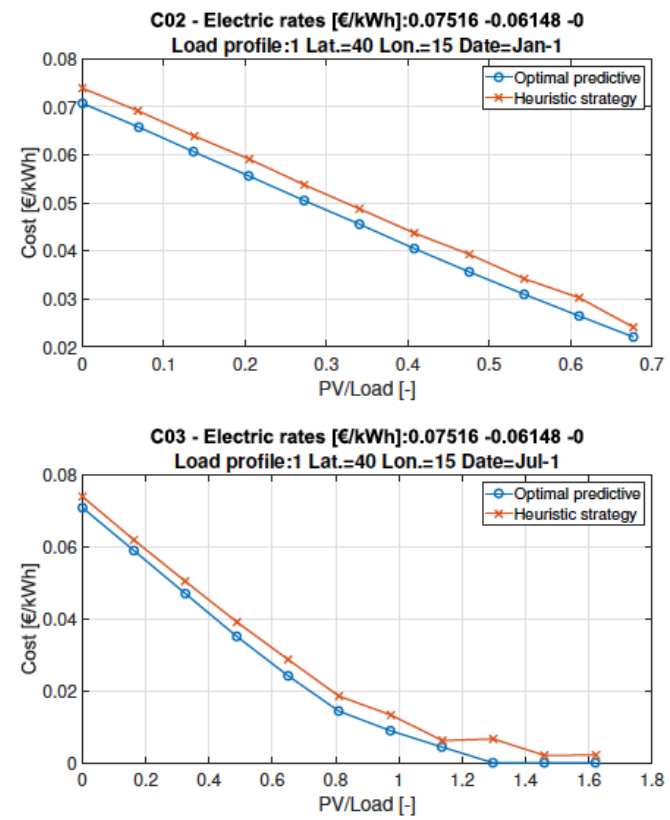

attery capacity/Load 
The cosine of the solar zenith angle $\vartheta_{z}$ is calculated as:

$\cos \vartheta_{z}=\cos L A T_{\text {loc }} \cos \delta \cos \omega+\sin \omega \sin \delta$

where $L A T_{l o c}, \delta$ and $\omega$ are the local geographical latitude, the solar declination and the hour angle, respectively.

The declination $\delta$ [rad] is computed as:

$\delta=0.4093 \sin \frac{2 \pi(284+n)}{365}$

The hour angle $\omega$ [rad] is given by:

$\omega=0.2618\left(t_{\text {solar }}-12\right)$

where $t_{\text {solar }}$ is the solar time, which is in turn computed as:

$t_{\text {solar }}=\frac{60 t_{l o c}+4\left(L O N_{s t d}+L O N_{l o c}\right)+E T}{60}$

where $t_{l o c}$ is the local time, $L O N_{\text {std }}$ and $L O N_{l o c}$ are the standard and local longitude, respectively, and ET is the so-called equation of time. The latter is calculated as:

$$
\begin{aligned}
E T & =229.2(0.000075+0.001868 \cos B-0.032077 \sin B \\
& -0.014615 \cos 2 B-0.04089 \sin 2 B) \\
& \text { with } B=\frac{(n-1) 2 \pi}{365}
\end{aligned}
$$

If a surface with a tilt angle $\beta$ [rad] and an azimuth angle $\gamma$ [rad] is considered, the cosine of the angle of incidence $\vartheta$ of solar irradiance is given by:

$$
\begin{aligned}
\cos \vartheta & =\sin \delta \sin L A T_{l o c} \cos \beta-\sin \delta \cos L A T_{l o c} \sin \beta \cos \gamma \\
& +\cos \delta \cos L A T_{l o c} \cos \beta \cos \omega+\cos \delta \sin L A T_{l o c} \sin \beta \cos \gamma \cos \omega \\
& +\cos \delta \sin \beta \sin \gamma \sin \omega
\end{aligned}
$$

When a tilted surface is considered, view factors with the sky and the ground can be introduced:

$$
\begin{aligned}
& F_{s k y}=\frac{1}{2}(1+\cos \beta) \\
& F_{g r o}=\frac{1}{2}(1-\cos \beta)
\end{aligned}
$$

The fraction of direct irradiance on a tilted surface can be computed after a correction factor $R B$ :
$R B=\frac{\cos \vartheta}{\cos \vartheta}$

The standard air mass $m_{s t d}$ has been computed from the Badescu model:

$$
\begin{gathered}
m_{\text {std }}=\frac{-\cos \vartheta_{z}+\sqrt{\cos ^{2} \vartheta_{z}+f^{2}-1}}{f-1} \\
\text { where } f=1+\frac{11}{6371.2}
\end{gathered}
$$

From the knowledge of $m_{\text {std }}$, the air mass $m$ computed at the altitude $h$ [m] of a considered site is given by:

$m=m_{\text {std }} \frac{p(h)}{p_{s t d}(h)}$

where $p(h)[\mathrm{Pa}]$ is the actual atmospheric pressure of the site and $p_{\text {std }}(h)$ is the standard pressure at height $h$ which can be computed as:

$p_{\text {std }}(h)=p_{\text {std }}(0)\left(\frac{T(h)}{T_{\text {std }}(0)}\right)^{5.2561}$

where $p_{\text {std }}(0)$ is the standard pressure at sea level (1 $017.085 \mathrm{hPa}), T(h)[\mathrm{K}]$ is the ambient temperature at altitude $h$, and $T_{\text {std }}(0)[\mathrm{K}]$ is the temperature at sea level which can be derived from $T(h)$ as:

$T_{\text {std }}(0)=T(h)+0.0065 h$

From the knowledge of the air relative humidity $u$ and of the air temperature at ground level $T[\mathrm{~K}]$, the thickness of precipitable water layer $u_{w}[\mathrm{~mm}]$ can be calculated:

$u_{w}=4.93 \frac{u}{T} \exp \left(26.23-\frac{5416}{T}\right)$

\section{REFERENCES}

[1] Alireza Akbari-Dibavar, Vahid Sohrabi Tabar, Saeid Ghassem Zadeh, and Ramin Nourollahi. Two-stage robust energy management of a hybrid charging station integrated with the photovoltaic system. International Journal of Hydrogen Energy, 2021; 46(24): 12701-12714. https://doi.org/10.1016/j.ijhydene.2021.01.127

[2] Gamal Alkawsi, Yahia Baashar, Dallatu Abbas U, Ammar Ahmed Alkahtani, Sieh Kiong Tiong, et al. Review of renewable energy-based charging infrastructure for electric vehicles. Applied Sciences, 11(9): 3847, 2021. https://doi.org/10.3390/app11093847

[3] Syed Muhammad Arif, Tek Tjing Lie, Boon Chong Seet, Soumia Ayyadi, and Kristian Jensen. Review of electric vehicle technologies, charging methods, standards and optimization techniques. Electronics, 2021; 10(16): 1910 https://doi.org/10.3390/electronics10161910 
[4] Viorel Badescu. Verification of some very simple clear and cloudy sky models to evaluate global solar irradiance. Solar Energy, 61(4): 251-264, 1997. https://doi.org/10.1016/S0038-092X(97)00057-1

Official Journal of the European Union. Regulation (EU) 2019/631 of the European Parliament and of the Council of 17 April 2019 setting CO2 emission performance standards for new passenger cars and for new light commercial vehicles, and repealing Regulations (EC) No 443/2009 and (EU) No 510/2011 (Text with EEA relevance.). Available online: https: /leur-lex.europa.eu/legalcontent/EN/TXT/?uri=celex: 32019R0631, 2021. Accessed on August 20th, 2021.

[6] Gestore dei Servizi Energetici. Valore del fattore emissivo relativo allâ $€^{\mathrm{TM}}$ energia elettrica fornita ai veicoli stradali a trazione elettrica. Technical report, Gestore dei Servizi Energetici, 2018.

[7] Anton Kersten, Artem Rodionov, Manuel Kuder, Thomas Hammarström, Anton Lesnicar, and Torbjörn Thiringer. Review of technical design and safety requirements for vehicle chargers and their infrastructure according to national swedish and harmonized european standards. Energies, 2021; 14(11): 3301. https://doi.org/10.3390/en14113301

[8] A.R. Kizhakkan, A. Awasthi, and A.K. Rathore. Mobility Management in Urban Areas: Models and Perspectives, Chapter 2: Electric Vehicle Charging Station Location Optimization. Nova, 2021.

[9] Karima Kouka, Abdelkarim Masmoudi, Achraf Abdelkafi, and Lotfi Krichen. Dynamic energy management of an electric vehicle charging station using photovoltaic power. Sustainable Energy, Grids and Networks, 2020; 24: 100402,. https://doi.org/10.1016/j.segan.2020.100402

[10] Desheng Li, Adama Zouma, Jian-Tang Liao, and Hong-Tzer Yang. An energy management strategy with renewable energy and energy storage system for a large electric vehicle charging station. eTransportation, 2020; 6: 100076. https://doi.org/10.1016/j.etran.2020.100076

[11] Guodong Liu, Madhu Sudhan Chinthavali, Suman Debnath, and Kevin Tomsovic. Optimal sizing of an electric vehicle charging station with integration of pv and energy storage. Technical report, Oak Ridge National Lab.(ORNL), Oak Ridge, TN (United States), 2021.
[12] Gianfranco Rizzo. Automotive applications of solar energy. IFAC Proceedings Volumes, 2010; 43(7): 174-185. https://doi.org/10.3182/20100712-3-DE-2013.00199

[13] Gianfranco Rizzo, Massimo Naddeo, and Cecilia Pisanti. Upgrading conventional cars to solar hybrid vehicles. International Journal of Powertrains, 7(1-3): 249-280, 2018. https://doi.org/10.1504/IJPT.2018.090352

[14] Scopus. Search Database. Available online: https: //www.scopus.com/search/form.uri?display=basic\#basic, 2021. Accessed on August 31st, 2021.

[15] Marco Sorrentino, Gianfranco Rizzo, and Luca Sorrentino. A study aimed at assessing the potential impact of vehicle electrification on grid infrastructure and road-traffic green house emissions. Applied Energy, 120: 31-40, 2014. https://doi.org/10.1016/j.apenergy.2014.01.040

[16] Baojun Sun. A multi-objective optimization model for fast electric vehicle charging stations with wind, pv power and energy storage. Journal of Cleaner Production, 2021; 288: 125564.

https://doi.org/10.1016/j.jclepro.2020.125564

[17] Francesco Antonio Tiano, Gianfranco Rizzo, and Dario Marra. Design and optimization of a charging station for electric vehicles based on compressed air energy storage. IFAC-PapersOnLine, 2018; 51(9): 230-235. https://doi.org/10.1016/j.ifacol.2018.07.038

[18] The Weather Channel. Weather Forecasts. Available online: https: //weather.com/, 2021. Accessed on August 20th, 2021.

[19] Meng Yang, Lihui Zhang, Zhenli Zhao, and Liwan Wang. Comprehensive benefits analysis of electric vehicle charging station integrated photovoltaic and energy storage. Journal of Cleaner Production, 2021; 302: 126967. https://doi.org/10.1016/j.jclepro.2021.126967

[20] Xiao Zhaoxia, Li Hui, Zhu Tianli, and Li Huaimin. Day-ahead optimal scheduling strategy of microgrid with evs charging station. In 2019 IEEE 10th International Symposium on Power Electronics for Distributed Generation Systems (PEDG), 2019; pages 774-780. IEEE. https://doi.org/10.1109/PEDG.2019.8807656

DOI: https://doi.org/10.31875/2410-2199.2021.08.9

(c) 2021 Rizzo et al.; Zeal Press.

This is an open access article licensed under the terms of the Creative Commons Attribution Non-Commercial License (http://creativecommons.org/licenses/by-nc/3.0/), which permits unrestricted, non-commercial use, distribution and reproduction in any medium, provided the work is properly cited. 